\title{
MORTALIDAD MATERNA EN EL INSTITUTO MATERNO INFANTIL, DE BOGOTA (1971 - 1973)
}

\author{
Dr. Fernando Sánchez Torres*
}

Con la política, cada vez más recomendada, de propiciar el "parto institucional" se busca otorgar la mayor protección posible a la grávida como también a su hijo por nacer. Pero para que tal objetivo se logre no basta que existan instituciones dedicadas a la atención materna e infantil sino que es menester además que el status sociocultural de las embarazadas alcance un nivel suficiente para que ellas mismas contribuyan a su protección. Como veremos en este estudio, muchos son los factores derivados de la ignorancia y la pobreza que inciden de manera notable sobre las tasas de mortalidad materna.

El Instituto Materno Infantil "Concepción Villaveces de Acosta", de Bogotá, desde su fundación en 1944, sigue siendo la maternidad más importante del país, no solo por el número de casos que atiende sino también por ser un hospital universitario, adscrito a la Facultad de Medicina de la Universidad Nacional. Por otra parte, es en el Distrito Especial de Bogotá el único centro asistencial destinado a atender las pacientes obstétricas y ginecológicas de escasos o nulos recursos económicos, es decir, aquella población femenina que no está protegida por ningún sistema convencional de salud (seguros sociales, cajas de previsión, etc.) y que no tiene po-

sibilidad de acceso a los servicios médicos privados. Lo anterior explica por qué el Instituto Materno Infantil atiende cerca del 35 por 100 de los nacimientos ocurridos anualmente en la capital del país, ciudad que cuenta con casi 3 millones de habitantes.

\section{Métodos}

Con el objeto de poder establecer claramente las causas de las muertes ginecobstétricas sucedidas en el Instituto, fue creado a fines de 1970 el Comité Permanente de Mortalidad Ginecobstétrica, compuesto por el Director Científico del Instituto, el Director del Departamento de Obstetricia y Ginecología, el Jefe de la Sección Obstétrica, el Jefe de la Sección Ginecológica, el Jefe del Servicio de Patología, y un representante de los médicos matriculados en los programas de postgrado (Cuadro № 1 ).

Acaecido un fallecimiento el médico que asistió de último a la enferma está obligado a comunicarlo enseguida por escrito a la Dirección del Instituto, en un formulario especial, y a solicitar la práctica de la respectiva au-

* Profesor Asociado del Departamento de Obstetricia y Ginecología (Universidad Nacional). Director Científico del Instituto Materno Infantil. 


\section{CUADRO NN 1}

\section{COMITE DE MORTALIDAD} GINECOBSTETRICA

- Director del Instituto

- Director del Dpto. de Cbst./Gin.

- Jefe de la Sección Obstétrica

- Jefe de la Sección Ginecológica

- Patólogo

- Representante de residentes

topsia. Una vez ejecutada esta y estudiacla la patología microscópica, el Comité se reúne para revisar detenidamente la historia clínica, citando si se cree necesario, a los médicos que participaron en el manejo de la enferma.

Adquirido un concepto integral del caso se conceptúa si la muerte era evitable o inevitable. Si era evitable se señalan los factores que influyeron en ella, tales como el institucional, el médico y el paciente. Este último no sólo abarca la circunstancia de que la enferma no hubiera acudido oportunamente al hospital, sino que también involucra los problemas derivados de su condición socio-cultural y que incidieron en la aparición de la enfermedad que la condujo a la muerte, por ejemplo, haber solicitado y consentido que se le provocara el aborto. Es verdad que este procedimiento, un tanto arbitrario, hace recaer en la paciente misma la culpabilidad de su fallecimiento, cuando muchas veces las fallas deben ser imputadas a la hostilidad del medio en que le tocó vivir. De todas maneras, juzgando así, con detenimiento y objetividad, cada defunción, es posible determinar las causas ciertas de muerte y señalar los factores que, directa $o$ indirectamente, intervinieron en ella.

A continuación presentamos los resultados del estudio de mortalidad materna llevado a cabo por el Comité durante 36 meses continuos, co- rrespondientes a los años 1971, 72 y 73.

Como definición de "muerte materna" hemos acogido la recomendada por la Federación Internacional de Sociedades de Ginecología y Obstetricia (FIGO) y la Asociación Médica Americana, y que a la letra dice: "Muerte Materna es la defunción de una mujer, por cualquier causa, durante el embarazo y hasta los 42 días después de la finalización de la gestación, sin tener en cuenta la edad del embarazo en el momento de la terminación o el método por el cual el mismo fue terminado". Por comprobar que con frecuencia surge duda acerca del grupo más apropiado para incluir algunas muertes que bien pueden corresponder a la vez a "causas obstétricas indirectas" y a "causas no obstétri-

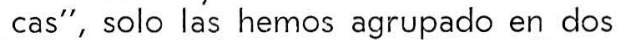
rubros: "obstétricas directas" y "otras causas".

\section{Material y Resultados}

\section{Tata de mortalidad materna}

Durante el lapso de nuestro estudio ccurrieron 68.930 nacimientos de niños vivos, y 209 muertes maternas, lo que arroja una tasa de mortalidad materna de 3.06 por 1.000. Es de anotar que mientras el número de nacidos vivos disminuyó en 1973, el número de defunciones maternas aumentó (Cuadro № 2). La explicación de este fenómeno en parte radica, como veremos adelante, en el aumento de los abortos provocados.

Al observar la curva de las tasas de mortalidad materna en los últimos diez años en el Instituto Materno Infantil, (Fig. 1), podemos apreciar que en vez de descender sostenidamente se elevó de manera brusca en el último año, pues mientras en 1964 fue de 2 por 1.000 en 1973 llegó a 3.9 por 1.000 , con alguna tendencia al descen- 


\section{CUADRO NN 2}

DEFUNCIONES MATERNAS (1971-73)

\begin{tabular}{lcccc}
\hline Año & 1971 & 1972 & 1973 & Total \\
\hline No nacidos vivos & 23.402 & 24.467 & 21.061 & 68.930 \\
Muertes maternas & 64 & 61 & 84 & 209 \\
Tasa de mortalidad & $2.73^{\circ} / 00$ & $2.49 \% / 00$ & $3.98 \% / 00$ & $3.06 \% / 00$ \\
\hline
\end{tabular}

INSTITUTO MATERNO-INFANTIL

TASAS DE MORTALIDAD MATERNA

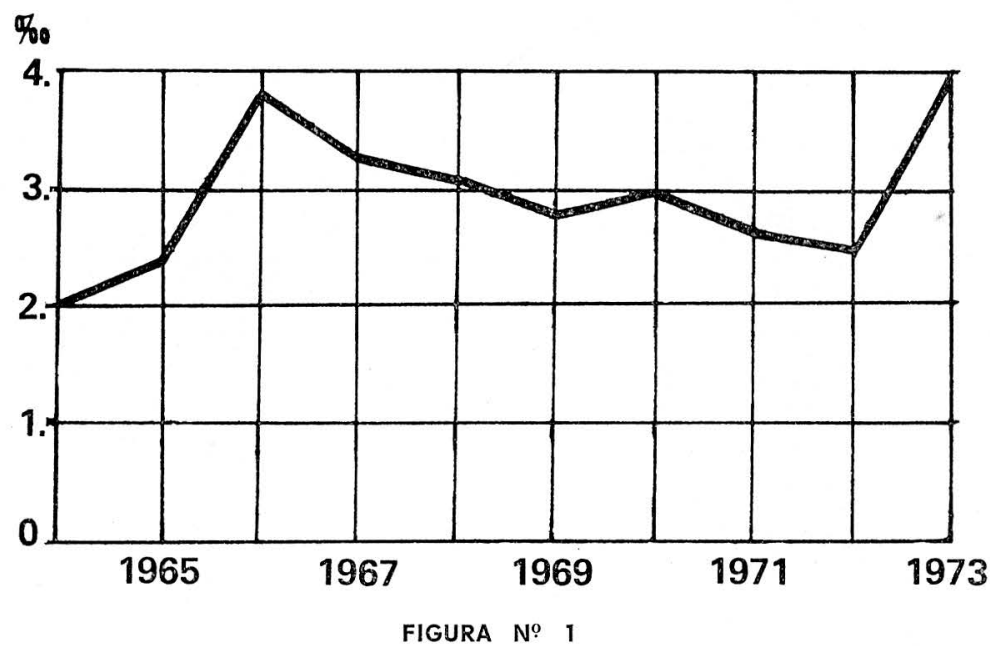

so, es cierto, descle 1966 hasta 1972. $\mathrm{Si}$ tenemos en cuenta que en los últimos años se han mejorado las condiciones locativas, se han actualizado las conductas y tratamientos y se ha incrementado la planta médica de especialistas, es decir, se han tecnificado los servicios, ha de aceptarse que los factores extrahospitalarios que inciden en la morbilidad y mortalidad maternas no solo persisten sino que también han aumentado.

\section{Autopsias}

Del total de muertes ginecobstétricas, 230, se practicaron 169 autopsias, es decir el 73 por 100. El Comité también se ocupó de las causas que impidieron adelantar los estudios postmortem, poniéndose de presente fallas institucionales y del personal médico, que han venido siendo subsanadas, lo cual asegura para el futuro un porcentaje mayor de necropsias (Cuadro № 3). Así y todo, consideramos que el número de autopsias fue aceptable. Insistimos, sí, que para hacer más fácil y efectiva la labor de un Comité de Mortalidad, debe, idealmente, estar complementada cada historia clínica con su respectivo informe de anatomía patológica. 
MORTALIDAD GINECOBSTETRICA: AUTOPSIAS

\begin{tabular}{|l|c|c|c||c|}
\hline Muertes & 68 & 68 & 96 & 232 \\
\hline Autopsias practicadas & 47 & 53 & 69 & $169(73 \%)$ \\
\hline No practicadas por: & & 1972 & 1973 & TOTAL \\
\hline Oposición Familiares & 5 & 5 & 11 & 21 \\
\hline Deficiencias Locativas & 12 & 2 & - & 14 \\
\hline Negligencia Patólogo & - & 4 & 8 & 12 \\
\hline Medicina Legal & 3 & 3 & 1 & 7 \\
\hline Historia Incompleta & 1 & 1 & 2 & 4 \\
\hline Exceso de trabajo & - & - & 5 & 5 \\
\hline
\end{tabular}

CUADRO № 3

\section{Causas de muerte materna}

Hemos mencionado atrás que para efecto de clasificar las muertes maternas solamente se han tenido en cuenta dos grandes grupos: el de "causas abstétricas directas" y el de "otras causas". No nos hemos ceñido a los tres grupos aceptados hasta ahora (obstétrica directa, obstétrica indirecta y no obstétrica) por considerar que los dos últimos no están claramente definidos, prestándose por ello a confusión. No es infrecuente observar en las publicaciones sobre el tema que unos autores incluyan en el grupo de "causa obstétrica indirecta", entidades que para otros tienen cabida en el de "causa no obstétrica", y viceversa. De ahí, también, que en el presente trabajo no establezcamos comparaciones con los resultados de otras instituciones, extranjeras o nacionales. Es necesario advertir, de otra parte, que los datos de mortalidad del presente trabajo no han sido depurados, vale decir que no se excluyó ningún caso a causa de la duración de la estancia antes del deceso, ni siquiera cuando la enferma no alcanzó a recibir atención alguna. En 83 casos la permanencia en el hospital fue menor de 24 horas, y entre estos en 5 fue menor de una hora. Lo anterior pone de presente la gravedad en que Ilegaron muchas de las enfermas.

\section{Muertes de causa obstétrica directa (Fig. 2)}

1. Infección. Esta sola entidad representa el 52 por 100 de las causas de mortalidad materna. De 109 falle- 


\section{INSTITUTO MATERNO-INFANTIL CAUSAS DE MORTALIDAD MATERNA 1971-73}

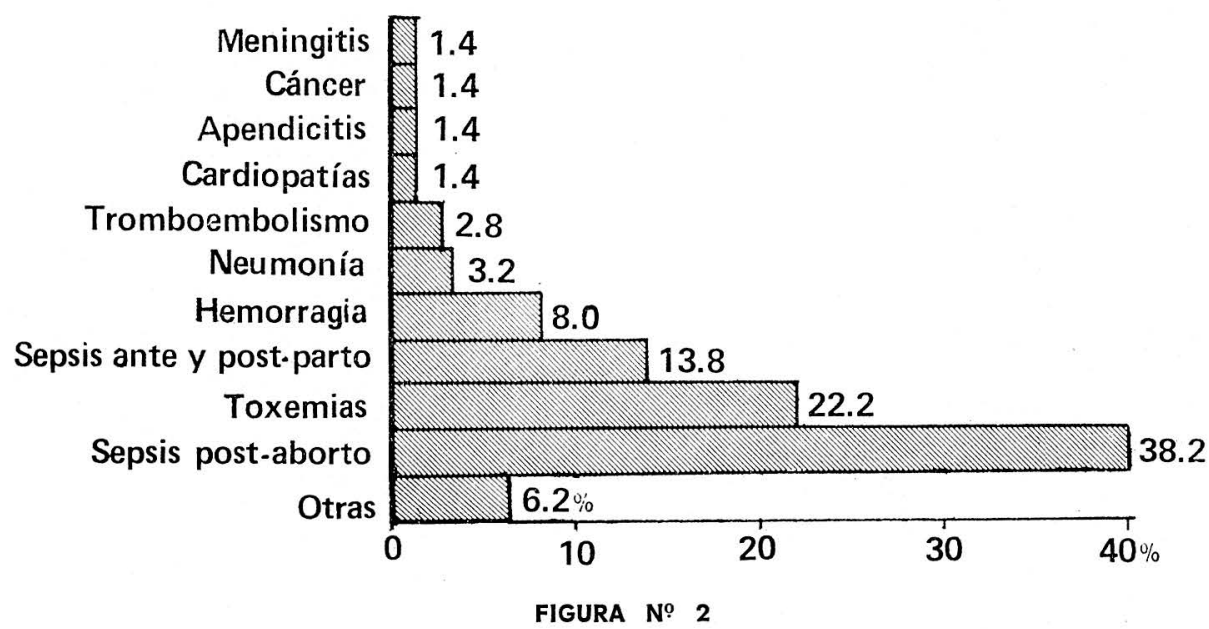

cimientos por sepsis, 80 correspondieron a sepsis post-aborto, 26 a sepsis puerperal ( 15 fueron partos vaginales y 11 intervenidos con cesárea) y 3 muertes ocurrieron antes del parto por infección amniótica.

2. Toxemias. La toxemia gravídica aguda, sola o asociada, fue la causa de muerte en 46 casos $(22,2 \%)$. En 32 de ellos las enfermas habían presentado convulsiones (eclampsia) y la complicación más frecuente fue el accidente cerebro vascular hemorrágico.

3. Hemorragia. El 8 por 100 de los fallecimientos ( 14 casos) se debieron a hemorragia por las siguientes causas: atonía uterina 7 , ruptura uterina 3, placenta previa 1, aborto 1, embarazo ectópico 1, ruptura de vena ovárica 1 .

4. Accidentes tromboembólicos. En 6 casos $(2,8 \%)$ se presentaron accidentes de este tipo, siendo 3 por embolia de líquido amniótico, 2 por em- bolia pulmonar y otro por trombosis de la arteria basilar.

5. Accidentes anestésicos. Tres mujeres fallecieron por esta causa.

\section{Muertes por otras causas (Fig. 2)}

A continuacicin presentamos una relación de las defunciones por causas distintas a las "obstétricas directas".

$\begin{array}{ll}\text { Neumonía } & 7 \\ \text { Cardiopatías } & 3 \\ \text { Apendicitis } & 3 \\ \text { Cáncer } & 3 \\ \text { Meningitis } & 3 \\ \text { Aneurismas } & 2 \\ \text { Amibiasis } & 2 \\ \text { Hepatitis viral } & 2 \\ \text { Intoxicación exógena } & 2 \\ \text { Púrpura trombocitopénica } & 1 \\ \text { Anemia drepanocítica } & 1 \\ \text { Paludismo } & 1 \\ \text { Herida cortopunzante } & 1\end{array}$

Como vemos, cerca del 15 por 100 de las muertes fueron de causa dife- 
rente a la obstétrica directa. De los 3 casos de cáncer registrados, uno era una leucemia linfoide crónica, otro un cáncer de seno y el tercero un cáncer de vejiga. Los 2 casos de intoxicación exógena lo fueron por ingestión voluntaria de fósforo blanco.

\section{Muertes evitables}

Como producto del estudio exhaustivo de cada caso el Comité de Mortalidad estableció que de las 209 defunciones maternas, $178(85 \%)$ eran evitables (Cuadro № 4). Puede pensarse que el criterio seguido para la calificación fue demasiado severo. Sin embargo, se hizo así con la finalidad de establecer hasta qué punto puede, idealmente, reducirse la mortalidad materna en el Instituto Materno Infantil. Por ejemplo, aceptamos como evitables las muertes consecutivas al aborto provocado (80 en total), a la infección intrahospitalaria, a la toxemia gravídica aguda, a los partos atendidos y complicados por comadronas, etc. Necesario es comentar también que el "factor médico" contribuyó de manera no despreciable a aumentar la tasa de mortalidad materna. Los descuidos en el examen y manejo de las enfermas, como también el llamado "error de juicio", estuvieron presentes en 48 casos. El factor "institución" estuvo implicado en 14 defunciones, por ausencia de equipos o elementos de tratamiento.

\section{MORTALIDAD MATERNA}

\section{$\begin{array}{lllll}\text { FACTOR } & 1971 & 1972 & 1973 & \text { TOTAL }\end{array}$}

\begin{tabular}{|l|c|c|c||c|}
\hline Paciente & 33 & 38 & 53 & 124 \\
\hline Médico & 12 & 9 & 11 & 32 \\
\hline Médico + Paciente & - & 2 & 6 & 8 \\
\hline Institución & 4 & - & 2 & 6 \\
\hline Institución + Médico & 1 & 1 & 4 & 6 \\
\hline Inst. + Méd. + Paciente & - & 1 & 1 & 2 \\
\hline
\end{tabular}

Muertes EVITABLES : 178

CUADRO NN 4

\section{Comentario}

Como balance del estudio adelantado por el Comité Permanente de Mortalidad Ginecobstétrica durante los últimos tres años en el Instituto Materno Infantil, puede concluirse que la mortalidad materna es demasiado alta, más aún tratándose de un hospital universitario, donde se presume que la atención médica debe ser de la más alta calidad. Empero, debe tenerse en cuenta que se trata también de una institución destinada a atender al grupo de población del más bajo nivel socio-cultural, aspecto este que, de manera inobjetable, conspira contra la salud de quienes lo constituyen.

Por otra parte, bien sabemos que el cuidado y vigilancia de la embarazada es de suma importancia para disminuir la mortalidad materna y fetal. Sin embargo, un altísimo porcentaje de las mujeres que acuden al Instituto Materno Infantil no han tenido nin- 
gún control médico en el curso de la etapa prenatal y solo demandan los servicios cuando se hallan en trabajo de parto o su gestación se ha complicado. Como producto de este abandono surgen graves problemas, que en otras circunstancias hubieran podido ser evitables o fácilmente corregibles.

Mención especial merece el problema del aborto provocado, no solo por ser la causa principal de mortalidad materna, sino también por constituirse en un pesado lastre para el exiguo presupuesto hospitalario. A pesar de que el Instituto cunta con un servicio destinado exclusivamente a la atención de pacientes con infección obstétrica, las muertes por complicaciones derivadas del aborto inducido han ido aumentando en forma alarmante. Mientras en 1971 fallecieron por esta causa 15 mujeres, en 1972 murieron 25 y 38 en 1973.

Aceptando que es posible corregir muchos de los factores que inciden en las defunciones maternas en el Instituto Materno Infantil, creemos, con algo de optimismo, que la tasa de mortalidad puede y debe fluctuar entre 0.5 y 1 por 1.000. Es natural que para que esto se logre debe sentirse con toda intensidad la acción del Estado, no solo mejorando y ampliando los programas de atención materna, sino también propiciando mejores condiciones socio-culturales para el gru- po humano que utiliza los servicios del Instituto. Asimismo, para disminuir los factores intrahospitalarios, se necesita intensificar la labor de auditoría médica. Creemos que para este efecto el Comité Permanente de Mortalidad se constituye en un instrumento de inmenso valor.

\section{Resumen}

Se presentan los resultados del estudio exhaustivo de 209 muertes maternas ocurridas en el Instituto Materno Infantil, de Bogotá, durante los años de 1971, 1972 y 1973.

La tasa, no depurada, de mortalidad materna observada en este lapso fue de 3.06 por 1.000 nacidos vivos.

El $85 \%$ de las defunciones fueron de causa obstétrica directa. La infección ocupó el primer lugar (52\%), a expensas particularmente del aborto provocado. La toxemia gravídica $22 \%$, la hemorragia $(8 \%)$ y los accidentes tromboembólicos $(2.8 \%)$ fueron las otras causas predominantes.

Se estableció, además, que cerca del $85 \%$ de las muertes eran evitables.

Se llama la atención acerca de los factores extrahospitalarios que inciden sobre la elevada tasa de mortalidad materna. 